\title{
User-centred design of flexible hypermedia for a mobile guide: reflections on the HyperAudio experience
}

\author{
Daniela Petrelli ${ }^{1}$ - Elena Not ${ }^{2}$ \\ ${ }^{1}$ Department of Information Studies, University of Sheffield, Regent Court, 211 Portobello street, \\ S1 4DP Sheffield, UK. E-mail: d.petrelli@shef.ac.uk \\ ${ }^{2}$ Cognitive and Communication Technology, ITC-irst, Trento, Italy.E-mail: not@itc.it
}

Published online: 19 May 2006

(c) Springer Science+Business Media B.V. 2006

Errors occurred in the above mentioned article which was published in User Modeling and User-Adapted Interaction 15(3-4): 303-338 (DOI 10.1007/s11257-005-8816-1).

On page 307, the second phrase should read:

Museums were chosen as a promising application test-bed because museum visitors move in the physical space looking for interesting exhibits and wishing to acquire information to deepen their knowledge and satisfy their interests.

On page 315, the first paragraph should read:

A survey was conducted to find out whether relations could be found between personal attributes (e.g. age, specific interests) and the way museums are visited. A questionnaire was organized around five topics:

On page 319, the ninth line should read:

Although some results from previous studies can be generalized and re-used, such as the suggestion of including maps or guided tours in museum mobile guides (Broadbend et al. 1998; Broadbend and Marti 1998), information that can influence adaptation needs to be collected anew by the new design team, and targeted toward the open questions that need direction.

On page 326, the second bulleted item should be changed to the one below:

Plug and play: adding or removing components should be easy, no matter whether they are already fully developed or still (hand-) simulated. Disconnection of modules in need of developer's attention or easy plugging-in of finished components is very important when a development team is involved.

On page 328 , the fourth sentence in the first bulleted item should be changed to the following: 
This feature is particularly important when the responsibility for creating the data belongs to authors who are domain experts, i.e. museum curators.

On page 329, the first paragraph should read:

The possibility of seeing at a glance an idea on, for example, the length of a presentation (i.e. the length of a path in the network) or the type of content delivered (e.g. anecdotal or historical) was very useful for creating a balanced network where all the nodes got a chance to be selected and listened to.

On page 330, the third from last sentence of the page should be changed to: Stereotypes are mentioned as a means for fast adaptation.

On page 332, the last sentence of the first paragraph should be changed to:

Finally, a new graphical interface was implemented to assist users in locating artworks by highlighting them on a 3D ego perspective reproduction of the room (Gabrielli et al. 1999). 